\title{
Theoretical Estimation of Vibrational Frequencies Involving Transition Metal Compounds
}

\author{
Thomas R. Cundari* and Philip D. Raby \\ Department of Chemistry, University of Memphis, Memphis, Tennessee 38152
}

Received: December 3, 1996; In Final Form: February 7, $1997^{\otimes}$

\begin{abstract}
The reliability of effective core potentials (ECPs) for estimating vibrational frequencies of transition metal (TM) complexes is assessed in relation to all-electron methods for main group compounds. Complexes with a multiple bond between a transition metal and chalcogen $(\mathrm{O}, \mathrm{S}$, or Se), chalcogenides, are investigated using the Stevens ECP/valence basis set scheme. Statistical treatment of the data indicates that ECPs, in addition to reliably modeling electronic structure, can be successful in estimating vibrational frequencies for TM complexes. As expected, theoretical prediction of vibrational data is not as accurate as the prediction of metric data for chalcogenides. However, agreement with experiment is still very good at the Hartree-Fock level of theory and is in even better accord upon the use of simple corrections to model well-known computational deficiencies (e.g., the neglect of anharmonic effects). Analysis of the data show interesting differences in predictive ability for first row transition metals versus second- and third-row analogues and oxo complexes versus their congeners with heavier chalcogens.
\end{abstract}

\section{Introduction}

It has become almost commonplace to see theoretical studies on the prediction of vibrational frequencies. ${ }^{1-7}$ The majority of this work has focused on organic compounds, with much less attention given to transition metal (TM) complexes. $^{8-11}$ Frenking and co-workers ${ }^{8}$ studied a small group of oxo and nitrido complexes, as well as $\mathrm{OsO}_{4-x} \mathrm{~F}_{2 x}(x=0-4)$ using Hartree-Fock methods. Sosa et al. ${ }^{9}$ investigated density functional theory (DFT) for prediction of geometries and vibrational frequencies of first- and second-row TM complexes. In a very recent and interesting paper, Russo et al. report an implementation of ECP analytic second derivatives, and applied it to the vibrational spectra of eight TM halides and oxohalides. ${ }^{10}$ These studies encouraged us to investigate more fully the ability of computational methods for estimation of vibrational data of a large and diverse series of TM complexes.

The present study examines the use of quantum methods to calculate harmonic vibrational frequencies for a family of transition metal complexes, i.e., chalcogenides $(\mathrm{TM}=\mathrm{Ch})$. Two corrections (scaling, linear) are evaluated for their efficacy in estimating experimental frequencies. Chalcogenides are particularly attractive for this research. These complexes are one of few families of TM complexes for which an extensive experimental database exists regarding both molecular structure and vibrational spectra. ${ }^{12-15}$ This provides the opportunity, perhaps unique among multiply bonded TM complexes, to compare theory and experiment with respect to vibrational spectra. Also, these complexes are of considerable interest in catalysis and advanced materials. The best catalysts and advanced materials intermediates are often the most active; hence, many important species are not stable enough, nor are they produced in sufficient concentrations, to be probed by powerful direct techniques such as X-ray crystallography. However, FTIR (Fourier transform infrared) and Raman spectroscopy can often be employed. ${ }^{12-15}$ Development of a simple, efficient scheme for accurate calculation of the vibrational spectra of $\mathrm{TM}=\mathrm{Ch}$ complexes is of interest for two main reasons. Such a scheme would be useful as an aid in identification of proposed reaction intermediates. Second,

* Author to whom correspondence should be addressed.

${ }^{\otimes}$ Abstract published in Advance ACS Abstracts, March 15, 1997. vibrational analysis is an effective probe of bonding. ${ }^{16}$ Combination of this data with complementary experimental and computational data can thus be used in the search for improved catalysts and advanced materials. A previous study showed the computational methods outlined below to be able to accurately predict the geometry of over 150 chalcogenides. ${ }^{17}$ This predictive capability for a large series of diverse complexes strongly suggests that the computational model is accurately describing the electronic structure of these complexes. Hence, it is reasonable to inquire whether the methods can be used to predict vibrational frequencies.

\section{Computational Methods}

The GAMESS ${ }^{18}$ program package was used for all calculations described herein. The effective core potentials and valence basis sets (VBSs) derived by Stevens et al. ${ }^{19}$ are used for all heavy atoms and the $-31 \mathrm{G}$ basis set for $\mathrm{H}$. ECPs replace the innermost core orbitals for transition metals and all core orbitals for main group elements. ${ }^{19}$ Thus, the $n \mathrm{~s}, n \mathrm{p}, n \mathrm{~d},(n+1) \mathrm{s}$, and $(n+1)$ p shells are treated explicitly for the d-block; for the main group, $n$ s and $n$ p shells are treated explicitly. ${ }^{19}$ In the standard implementation, transition metal (TM) valence basis sets are quadruple- $\zeta$ and triple- $\zeta$ for sp and d shells, respectively, while main group (MG) elements have a double- $\zeta$-plus-polarization VBS. This ECP/VBS combination is termed SBK(d).

Geometries are optimized at the restricted Hartree-Fock (RHF) and restricted open-shell Hartree-Fock (ROHF) level for closed- and open-shell systems, ${ }^{17}$ respectively, before calculating the energy Hessian. Previous research compared geometries calculated with RHF and Møller-Plesset secondorder perturbation theory (MP2) wave functions using the SBK(d) scheme. ${ }^{20}$ This study of multiply bonded complexes, chalcogenides included, led to two important conclusions. First, both RHF/SBK(d) and MP2/SBK(d) geometries are close to experimental data. If anything, $\mathrm{MP} 2 / \mathrm{SBK}(\mathrm{d})$ tends to overcorrect differences between $\mathrm{RHF} / \mathrm{SBK}(\mathrm{d})$ and experimental geometries. $^{20}$ Second and more importantly, there is only a small difference in calculated geometries despite the much greater expense of MP2 calculations. To summarize, TMelement bonds differ by $1-3 \%$ with bond angles involving the TM being, on average, within 3-4\% of each other at the RHF/ 
SBK(d) and MP2/SBK(d) levels of theory. ${ }^{20}$ Correlation effects are generally more significant toward the right in a TM series, toward the left in a MG row, and for heavier elements in a main group column. ${ }^{20}$ These results, along with the accuracy of structural prediction, strongly supports the suitability of RHF/ SBK(d) wave functions for these systems. ${ }^{17,20}$

Vibrational frequencies are calculated by numerical differentiation (single differencing) of analytical gradients for optimized geometries and are based on the harmonic approximation. The capability to calculate ECP analytic second derivatives does not yet exist in GAMESS, although previous studies suggest that the differences between analytic and numerical differentiation ${ }^{3,4}$ are well within the experimental uncertainties of vibrational frequencies of most TM complexes. Frequencies derived by ECP-based computations are compared with experimental values in three different ways: (1) raw (i.e., calculated using the harmonic approximation), (2) scaled, and (3) by means of a linear, least-squares correction. The reasoning for examining different treatments of the theoretical data is discussed more fully in the following section.

Agreement between computed harmonic frequencies and experimental frequencies (which include anharmonic effects) is assessed using several statistical approaches. These are as follows.

1. Range of Absolute Differences. The range of absolute difference quantity shows the minimum and maximum absolute differences between calculated $\left(v_{\text {ecp }}\right)$ and experimental $\left(v_{\exp }\right)$ frequencies.

$$
\left|v_{\text {ecp }}-v_{\text {exp }}\right|=|\Delta|
$$

2. Mean Absolute Difference. The mean absolute difference is the average of the absolute value differences between calculated and experimental frequencies $(N=$ total number of frequencies).

$$
\left[\sum_{i=1}^{N}\left|\Delta_{i}\right|\right] / N=\Delta_{\text {ave }}
$$

3. Mean Percent Difference. The mean percent difference is the average difference between the calculated and experimental frequencies in terms of a positive or negative percentage.

$$
100\left[\sum_{i=1}^{N}\left(\Delta_{i} / \nu_{\exp }\right)\right] / N=\% \Delta_{\text {ave }}
$$

\section{Results and Discussion}

1. The Transition Metal-Chalcogenide Data Set. Initial fits were done with all chalcogenides studied in this research. A plot of calculated versus experimental stretching frequencies is given in Figure 1. It is clear from Figure 1 that several compounds are widely distinct from the majority which show good linear correlation between calculated harmonic and experimental frequencies. These divergent compounds fall into one of two groups, i.e., metallates with a charge of -4 (titanate, zirconate, and hafnate) and $\mathrm{d}^{2}$-tetraoxos. Figure 2 shows a plot comparing theoretical and experimental results with these families of compounds excluded. The mean absolute difference between theoretical and experimental $\Delta_{\text {ave }}$ (see eq 2), is 120 $\mathrm{cm}^{-1}$ for the data in Figure $1 ; \Delta_{\text {ave }}$ improves to $112 \mathrm{~cm}^{-1}$ upon removal of tetraanionic and $\mathrm{d}^{2}$-tetraoxo complexes. More importantly, the linearity of the theoretical-experimental correlation improves significantly upon removal of these two groups of complexes. For the entire linearly corrected data set, $\Delta_{\text {ave }}$ $=54 \mathrm{~cm}^{-1}$, while $\Delta_{\text {ave }}$ is $38 \mathrm{~cm}^{-1}$ upon removal of tetraanionic

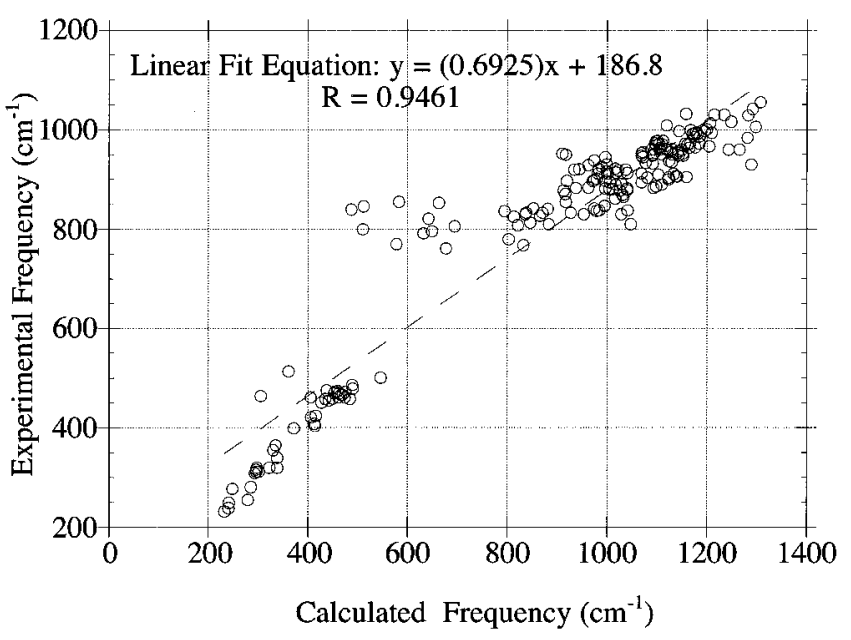

Figure 1. Plot of calculated harmonic versus experimental frequencies (in $\mathrm{cm}^{-1}$ ) for entire data set including $\mathrm{d}^{2}$-metallates and tetraanionic metallates. The least-squares linear fit of the data is also given.

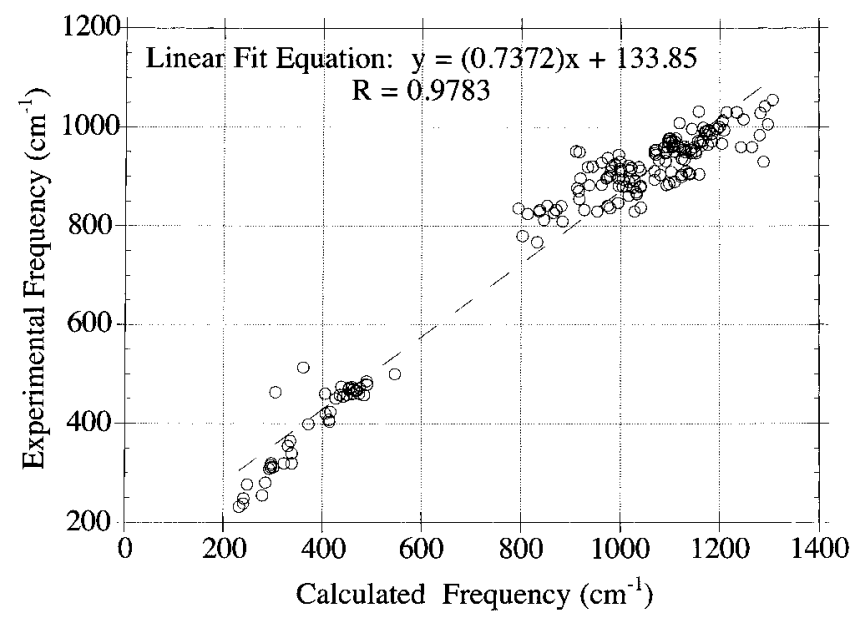

Figure 2. Plot of calculated harmonic versus experimental frequencies (in $\mathrm{cm}^{-1}$ ) for entire data set excluding $\mathrm{d}^{2}$-metallates and tetraanionic metallates. The least-squares linear fit of the data is also given.

and $\mathrm{d}^{2}$-tetraoxo complexes. For $\mathrm{d}^{2}$-tetraoxos, poor agreement may be due to problems in the Hartree-Fock wave function when the symmetry of the $\mathrm{e}^{2}$ ground state of the $T_{\mathrm{d}}$ molecule is reduced for numerical calculation of the energy Hessian. For tetraoxo tetraanions it seems plausible that poor behavior is due to the large negative charge making environmental effects have an appreciable effect on the experimental stretching frequencies (which were measured in the solid state) ${ }^{21}$ Electron correlation and diffuse basis functions (particularly on oxygen) may be especially critical for very highly charged anions. The following analyses exclude $\left[\mathrm{MO}_{4}\right]^{4-}$ and the $\mathrm{d}^{2}-\left[\mathrm{MO}_{4}\right]^{\mathrm{q}-}$.

A set of 93 transition metal chalcogenides $(\mathrm{TM}=\mathrm{Ch})$ and 184 $\mathrm{TM}=\mathrm{Ch}$ stretching frequencies has been used to compare RHF/ $\mathrm{SBK}(\mathrm{d})$ versus experimental vibrational frequencies found in the literature. ${ }^{12-15}$ The data set includes 71 oxo, 6 sulfide, 5 selenide, and 6 oxo(sulfide) complexes and 1 oxo(telluride) complex and 4 oxo(sulfide)(selenide) complexes. Although tellurides have been structurally characterized, ${ }^{2-25}$ no TMtelluride stretching frequencies have been reported. The structural motifs studied, Figure 3, are the most important in $\mathrm{TM}=\mathrm{Ch}$ chemistry. In this section we seek to compare the present results for transition metal complexes with that reported by Pople et al. ${ }^{2}$ for main group compounds. One difference between this study and that by Pople et al. ${ }^{2}$ is that degenerate modes (e.g., the $t_{2}$ stretch in tetrahedral metallates, $\left[\mathrm{M}(\mathrm{Ch})_{4}\right]^{\mathrm{q}^{-}}$) have not been multiple times counted in the present analyses. 


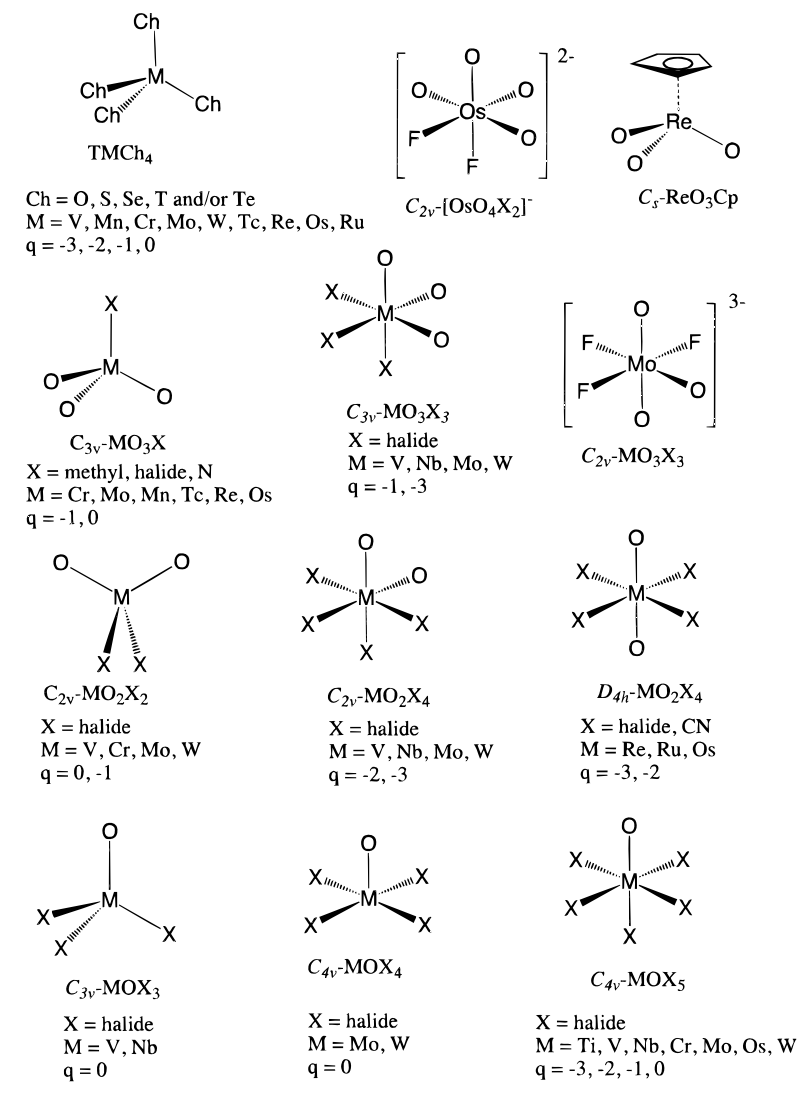

Figure 3. Transition metal chalcogenide $(\mathrm{TM}=\mathrm{Ch})$ structural motifs investigated. A complete listing of calculated harmonic and experimental stretching frequencies is given in the Supporting Information.

We have focused on $\mathrm{TM}=\mathrm{Ch}$ stretching frequencies since experimental assignment of these modes is the most accurate and least ambiguous. Also, $\mathrm{TM}=\mathrm{Ch}$ stretching frequencies are of greatest interest in connection with coupling vibrational data with electronic and molecular structural data ${ }^{17,24}$ for probing the nature of the TM-chalcogen bond.

2. Theoretical Approaches. The calculation of harmonic vibrational frequencies is less computationally intensive, regardless of wave function type used, than calculations which include anharmonic effects. Additionally, after extensive use, the systematic errors introduced by using the harmonic approximation are well-known for MG compounds. ${ }^{1-6}$ Calculated harmonic frequencies are typically corrected to experimental values through the use of scaling factors $(\lambda)$, eq 4 . Various

$$
v_{\text {scaled }}=\lambda \nu_{\text {calcd }}
$$

researchers ${ }^{1-6}$ have recommended values of $\lambda \approx 0.9-1.0$ for predictive purposes when using theoretical frequencies for main group compounds determined with different basis sets and levels of theory.

In the present research, we look at scaling (eq 4) as well as an alternative approach employing a linear least-squares fit, eq 5 , for obtaining closer agreement between calculated and

$$
v_{\text {linear }}=\lambda^{\prime} v_{\text {calcd }}+b
$$

experimental $\mathrm{TM}=\mathrm{Ch}$ vibrational frequencies. The addition of an extra parameter yields more flexibility for the linear fit. It will be shown that for $\mathrm{TM}=\mathrm{Ch}$ complexes, unlike their main group counterparts, a linear fit gives much better predictive capacity versus scaling. A comparison of RHF/SBK(d) and experimental frequencies is presented below. The viability of this method using ECPs for TM complexes is considered in reference to Pople's ${ }^{2}$ all-electron, main group study.

3. Comparison of Main Group and Transition Metal Results. The scaling and linear fit results for $\mathrm{TM}=\mathrm{Ch}$ (calculated with the RHF/SBK(d) scheme for $\mathrm{TM}=\mathrm{Ch}$ stretching modes) and MG (calculated via all-electron RHF/6-31G* methods for stretching, bending, etc., modes) harmonic vibrational frequencies are collected in Table 1.

3.1. Main Group, All-Electron Results. The absolute values of differences $|\Delta|$ between experimental and all-electron (HF/6-31G*) calculations ${ }^{2}$ in the main group data range from $2 \mathrm{~cm}^{-1}$ (three modes) - $566 \mathrm{~cm}^{-1}\left(4 \mathrm{a}^{\prime}\right.$ mode of $\left.\mathrm{NCl}_{2} \mathrm{~F}\right)$. Scaling all-electron ( $v_{\mathrm{ae}}$ in eqs 6 and 7 ) frequencies, ${ }^{2}$ using the scaling

$$
\begin{gathered}
v_{\text {scaled }}=0.8949 v_{\mathrm{ae}} \\
v_{\text {linear }}=\left(0.9020 v_{\mathrm{ae}}\right)-16.094
\end{gathered}
$$

factor of 0.8949 , (eq 6 ) decreases the range of $|\Delta|$ from $0 \mathrm{~cm}^{-1}$ (seven modes) to $420 \mathrm{~cm}^{-1}\left(4 \mathrm{a}^{\prime}\right.$ mode of $\left.\mathrm{NCl}_{2} \mathrm{~F}\right)$. A linear fit, eq 7 , of the main group data versus experimental results does not significantly decrease the range of $|\Delta|: 0 \mathrm{~cm}^{-1}$ (9 modes)$414 \mathrm{~cm}^{-1}$ ( $4 \mathrm{a}^{\prime}$ mode of $\mathrm{NCl}_{2} \mathrm{~F}$ ).

3.2. Transition Metal, Effective Core Potential Results. Comparison of unscaled RHF/SBK(d) stretching frequencies versus experimental results for $\mathrm{TM}=\mathrm{Ch}$ shows that the absolute value of the difference between theoretical and experimental frequencies range from $0 \mathrm{~cm}^{-1}(3$ modes $)$ to $359 \mathrm{~cm}^{-1}$ $\left(\left[\mathrm{CrOCl}_{5}\right]^{2-}\right)$. Analysis of the $\mathrm{TM}=\mathrm{Ch}$ data, eq 8 and Table 1 ,

$$
v_{\text {scaled }}=0.8695 v_{\text {ecp }}
$$

shows RHF/SBK(d) frequencies to be systematically high by an average of $10 \%$. Thus, RHF/SBK(d) harmonic frequencies for the TM complexes display the same systematic overestimation, and of similar magnitude, of experimental frequencies as do more well-studied MG compounds. This suggests that similar correction approaches for $\mathrm{TM}$ complexes and $\mathrm{MG}$ compounds can be used.

Use of the optimal scaling factor (0.8695 in eq 8) decreases the range of absolute differences between calculation and experiment by over $40 \%$ : $0 \mathrm{~cm}^{-1}\left(\left[\mathrm{NbOCl}_{5}\right]^{2-}\right)-200 \mathrm{~cm}^{-1}$ $\left(\left[\mathrm{NbO}_{2} \mathrm{~S}_{2}\right]^{2-}\right)$. Another approach to improving theoreticalexperimental agreement is to use a linear fit, eq 9 and Figure 2,

$$
v_{\text {linear }}=\left(0.7372 v_{\text {ecp }}\right)+133.85
$$

for the $\mathrm{TM}=\mathrm{Ch}$ data. This gives an even greater improvement in the range of $|\Delta|$ (eq 2): $0 \mathrm{~cm}^{-1}\left(\mathrm{TcO}_{3} \mathrm{~F}\right)-154 \mathrm{~cm}^{-1}$ $\left(\left[\mathrm{CrOCl}_{5}\right]^{2-}\right)$. The range of $|\Delta|$ in the transition metal cases is approximately half that observed for main group, all-electron results. ${ }^{2}$ Presumably, this is partly a function of the wider range of experimental frequencies $\left(4000 \mathrm{~cm}^{-1}\right)$ for MG compounds as opposed to $\mathrm{TM}=\mathrm{Ch}$ complexes $\left(1000 \mathrm{~cm}^{-1}\right)$. One interesting difference is that the agreement does not seem to improve for the main group compounds upon going from a scaling to linear correction, Table 1: the range of $|\Delta|$ decreases by only $6 \mathrm{~cm}^{-1}$ for the RHF/6-31G*, MG results and by $46 \mathrm{~cm}^{-1}$ for the RHF/SBK(d), transition metal results.

The mean absolute difference $\Delta_{\text {ave }}$ for calculated $\mathrm{TM}=\mathrm{Ch}$ versus experimental frequencies is $112 \mathrm{~cm}^{-1}$. Scaling of the theoretical results, using the 0.8695 factor, more than halves $\Delta_{\text {ave }}$ to $54 \mathrm{~cm}^{-1}$, Table 1 . Use of the linear fit equation (eq 9) gives further reduction of $\Delta_{\text {ave }}$ to $38 \mathrm{~cm}^{-1}$. In comparison, $\Delta_{\text {ave }}$ for Pople's calculated frequencies on main group complexes was $172 \mathrm{~cm}^{-1}$. Scaling of the data by Pople et al., ${ }^{2}$ using their recommended 0.8949 scaling factor, improves $\Delta_{\text {ave }}$ by a factor 
TABLE 1: Statistical Analysis of TM-Chalcogenide Frequency Prediction

\begin{tabular}{|c|c|c|c|c|c|c|}
\hline \multirow[b]{2}{*}{ parameter $^{c}$} & \multicolumn{3}{|c|}{ TM-chalcogens ${ }^{a}$} & \multicolumn{3}{|c|}{ MG compnds ${ }^{b}$} \\
\hline & raw & scaled & linear & raw & scaled & linear \\
\hline$\lambda$ & & 0.8695 & 0.7372 & & 0.8949 & 0.9020 \\
\hline$b$ & & & 133.85 & & & -16.09 \\
\hline$|\Delta|$, range of absolute difference $\left(\mathrm{cm}^{-1}\right)$ & $0-359$ & $0-200$ & $0-154$ & $2-566$ & $0-420$ & $0-414$ \\
\hline$\Delta_{\text {ave }}$, mean absolute difference $\left(\mathrm{cm}^{-1}\right)$ & 112 & 54 & 38 & 172 & 34 & 34 \\
\hline$\% \Delta_{\text {ave }}$, mean percent difference & 10 & -4 & 1 & 12 & 1 & -1 \\
\hline
\end{tabular}

${ }^{a}$ Experimental vibrational frequencies for transition metal chalcogenides were obtained from the literature. ${ }^{12-15}$ See Figure 2 for optimal linear least-squares fitting of data. ${ }^{b}$ Main group raw data and the optimal scaling factor $(\lambda)$ were obtained from the data in ref $2 .{ }^{c}$ Parameters are defined in eqs $1-3$.

TABLE 2: Statistical Analysis as a Function of Metal

\begin{tabular}{|c|c|c|c|c|c|}
\hline group IV & group V & group VI & group VII & group VIII & row $^{c}$ \\
\hline $\operatorname{Ti} 98,{ }^{a}(1)^{b}$ & $\begin{array}{l}\text { V 38, (16) } \\
\text { Nb 59, (14) } \\
\text { Ta 40, (4) }\end{array}$ & $\begin{array}{l}\text { Cr 55, (13) } \\
\text { Mo 25, (43) } \\
\text { W 33, (40) }\end{array}$ & $\begin{array}{l}\operatorname{Mn} 93,(6) \\
\operatorname{Tc} 21,(6) \\
\operatorname{Re} 26,(21)\end{array}$ & $\begin{array}{l}\mathrm{Ru} 57,(4) \\
\text { Os 44, (16) }\end{array}$ & $\begin{array}{l}55,(36) \\
33,(67) \\
34,(81)\end{array}$ \\
\hline $98,(1)$ & $47,(34)$ & $32,(96)$ & $37,(33)$ & $47,(20)$ & \\
\hline
\end{tabular}

${ }^{a}$ All chalcogenide stretching frequencies, linearly corrected using eq 9 , are used to calculate the average absolute frequency differences $\left(\Delta_{\text {ave }}\right.$, $\mathrm{cm}^{-1}$ ) as a function of transition metal. ${ }^{b}$ Number of frequencies used to compute the average. ${ }^{c}$ This is $\Delta_{\text {ave }}$ (in $\mathrm{cm}^{-1}$ ) for each row in the transition series. ${ }^{d}$ This is $\Delta_{\text {ave }}\left(\right.$ in $\mathrm{cm}^{-1}$ ) for each transition metal triad.

of 5 to $34 \mathrm{~cm}^{-1}$, while a linear fit approach also yields $\Delta_{\text {ave }}=$ $34 \mathrm{~cm}^{-1}$ for the main group data, Table 1. Thus, the overall quality of the RHF/SBK(d) scheme is comparable to that seen for the more well-studied main group systems using 6-31G* all-electron methods. ${ }^{2}$

4. Specific Trends in TM-Chalcogenides. The previous discussion viewed the TM-chalcogenide frequency calculations in an overall sense and compared it with a previous study ${ }^{2}$ employing all-electron calculations on main group compounds. In the following sections we wish to use the data to explore specific trends which emerge from examination of $\mathrm{TM}=\mathrm{Ch}$ frequencies. It is hoped that analysis of these trends will give some insight into this important family of complexes as well as provide the necessary working parameters for others who may wish to employ the results of this research. The trends are discussed in terms of (1) how well the calculation has performed in relation to the experimental data, (2) highlighting various chemical moieties which seem less/more amenable to the methodology, and (3) offering, where possible, some basis for specific trends.

4.1. Differences as a Function of Metal. It is plausible that a trend in frequency differences (theoretical versus experimental) could exist as a function of metal, given the differences in chemical bonding which can occur both within a triad and along a row of the transition series. ${ }^{26}$ Therefore, $\Delta_{\text {ave }}$ of the linearly fitted calculated versus experimental frequencies were examined for the following trends: (1) by row and (2) by periodic table group. Twelve different transition metals are represented in the data set, Table 2.

The chalcogenide data set was examined for a transition metal-related trend as a function of periodic table group. The $\Delta_{\text {ave }}$ (linearly corrected theory versus experimental frequencies) are 98 (one frequency), 47, 32, 37, and 47, respectively, for periodic groups IV-VIII. Thus, the best predictive ability is obtained for chalcogenides of the chromium triad metals which comprise the largest subset of the data, with only slightly less agreement for the metals of the manganese triad, Table 2. One must be careful in analyzing the data, since it becomes increasingly sparse as one moves away from the center of the transition series. However, if there is any trend in $\Delta_{\text {ave }}$ it is that the best theoretical-experimental agreement is obtained for TMs toward the center of the series (chromium and manganese triads) and diminishes as one moves to the right

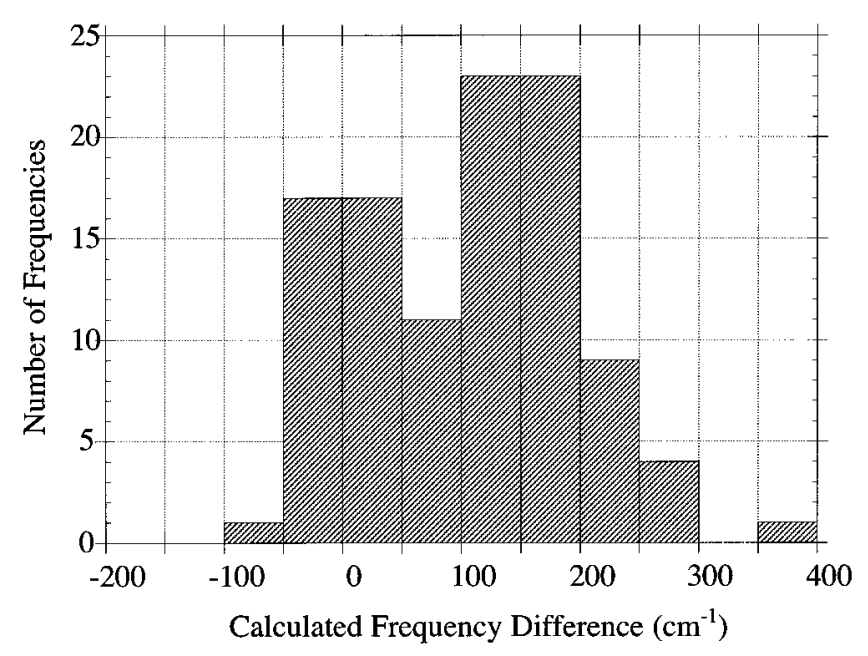

Figure 4. Histogram showing the differences (in $\mathrm{cm}^{-1}$ ) between calculated harmonic (without either linear or scaling correction) and experimental frequencies.

(toward earlier, oxophilic TMs) and the left (toward the later transition metals).

Table 2 also gives $\Delta_{\text {ave }}$ as a function of transition series row. Averaging across each periodic table row shows a significantly higher $\Delta_{\text {ave }}$ for first-row transition metals $\left(55 \mathrm{~cm}^{-1}\right)$ as compared with second- and third-row TMs $\left(33 \mathrm{~cm}^{-1}\right.$ and $34 \mathrm{~cm}^{-1}$, respectively). All $\mathrm{TM}-$ chalcogenide stretching frequencies are used for this comparison. A comparison using only TM-oxo stretches gives the same trend. The similar results for complexes of the second- and third-row complexes are in line with the similar chemistry of these metals. ${ }^{26}$ Of greater interest is the comparison involving first-row TMs versus their second- and third-row congeners. The bonding in the first transition series is more ionic/dative due to the contracted nature of the $3 \mathrm{~d}$ orbitals. The more radially expansive $4 \mathrm{~d}$ and $5 \mathrm{~d}$ enhance overlap with ligand-based orbitals, and hence yield greater covalent bonding for complexes of second- and third-row transition metals.

4.2. Light versus Heavy Chalcogenides. An interesting fact which is obvious from Figure 4 is the bimodal distribution for the differences between calculated harmonic frequencies and experimental values for $\mathrm{TM}=\mathrm{Ch}$. One of the peaks (centered around $0 \mathrm{~cm}^{-1}$ ) is largely comprised of heavier chalcogenides 
$\left(\mathrm{Ch}=\mathrm{S}\right.$ or Se), while the other peak (centered around $\left.150 \mathrm{~cm}^{-1}\right)$ is comprised largely of oxos $(\mathrm{Ch}=\mathrm{O})$. An analysis of $\mathrm{TM}-$ oxo frequencies reveals $\Delta_{\text {ave }}=141 \mathrm{~cm}^{-1}$ for uncorrected frequencies. Heavier chalcogenides have $\Delta_{\text {ave }}=22 \mathrm{~cm}^{-1}$, indicating the calculated frequencies to be in much closer agreement with experiment than for oxos. There is no experimental data which would allow us to confirm or deny whether there is a significant difference in the anharmonicity of oxos as compared to heavier congeners. It is, of course, possible that the behavior of heavier chalcogenides may arise from cancelation of errors such as anharmonicity which tends to overestimate frequencies and bonds which are too long (which would lead to lower than expected frequencies). The latter point, however, is not supported by extensive structural studies of TM-chalcogenides ${ }^{17,20}$ which showed the RHF/SBK(d) scheme to give very accurate estimation of structural data for oxos, sulfides and selenides and a relatively small electron correlation effect on $\mathrm{TM}=\mathrm{Ch}$ geometries. ${ }^{20}$

The TM-oxos are amenable to use of curve-fitting corrections (scaling/linear least-squares fit) to give closer theoreticalexperimental agreement. Scaling of calculated TM-oxo stretching frequencies moved $\Delta_{\text {ave }}$ from 141 to $50 \mathrm{~cm}^{-1}$; linear correction of TM-oxo frequencies gave a further improvement in $\Delta_{\text {ave }}\left(40 \mathrm{~cm}^{-1}\right)$. However, scaling of TM-sulfide and TMselenide data increases $\Delta_{\text {ave }}$ from 22 to $66 \mathrm{~cm}^{-1}$, whereas linear fitting also gives a slight increase in $\Delta_{\text {ave }}$ to $30 \mathrm{~cm}^{-1}$. The optimal scaling factor for TM-oxo stretches is $\lambda=0.86$, whereas the scaling factor for heavier congeners is $\lambda \approx 1.0$. Since the data is dominated by oxos, application of a linear correction to the entire data set will tend to overcorrect frequencies for heavier chalcogenides.

The different behavior between TM-oxos and their heavier analogues is very interesting in connection with previous experimental and computational studies. ${ }^{17,24 a}$ Theoretical and experimental ${ }^{17,24 \mathrm{a}}$ studies of $\mathrm{Ti}$-triad chalcogenides suggest that

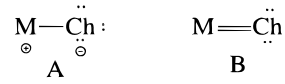

oxos have a greater contribution from a single-bonded structure A, while heavier analogues are more heavily weighted toward the double-bonded structure B. A fundamental difference in the nature of the TM-oxo multiple bond versus the bonding of the heavier analogues could explain the dichotomy between the two groups of stretching frequency calculations.

\section{Summary and Conclusions}

The primary goal of this research was to assess the reliability of effective core potentials (ECPs) for estimating vibrational frequencies of transition metal (TM) complexes in relation to all-electron methods for main group compounds. Specific trends which emerged were also explored. The effect of the chalcogenide ligand (oxo versus heavier congeners) was discussed along with the influence of the transition metal. Several conclusions have resulted from this research, the most important of which are summarized below.

1. First and most importantly, the use of ECPs for transition metal complexes gives a degree of accuracy in the prediction of vibrational frequencies comparable to more well-tested allelectron methods for main group compounds. ${ }^{1-7}$ The SBK(d) scheme $^{19}$ was used in this research since a previous study showed it to be highly accurate for prediction of metric data of $\mathrm{TM}=$ Ch complexes. ${ }^{17}$ Other ECP implementations with similar valence basis sets will most likely do as well. ${ }^{8,10,27}$ Linearly fitted SBK(d) calculations for the entire TM-chalcogenide data set gave an overall absolute difference of $38 \mathrm{~cm}^{-1}$ (theory versus experiment), whereas a linear fit of main group data ${ }^{2}$ gave an absolute difference of $34 \mathrm{~cm}^{-1}$. It should be noted that experimental frequencies for many TM complexes often have a range of values (as much as $20-30 \mathrm{~cm}^{-1}$ ) due to measurements in different phases, different solvents, or experimental error. ${ }^{12-16}$ The above treatments of the data clearly indicate that ECP methods, in addition to reliably modeling electronic structure,${ }^{27}$ can be successful in estimating vibrational frequencies for TM complexes.

2. One interesting difference between TM and MG results is that agreement between theory and experiment does not improve for MG compounds upon going from a scaling (eq 8) to linear (eq 9) correction. In other words, systematic errors encountered by using the harmonic approximation are more consistent throughout the main group series.

3. For linearly corrected harmonic frequencies, there is a significantly higher average absolute frequency difference for first-row transition metals $\left(55 \mathrm{~cm}^{-1}\right)$ as compared with secondand third-row transition metals (33 and $34 \mathrm{~cm}^{-1}$, respectively). All TM-chalcogenide stretching frequencies were used for this comparison. A comparison using only TM-oxo stretches gives the same trend.

4. The optimal scaling factor for oxos $(\lambda \approx 0.86)$ is different from that for heavier chalcogenides $(\lambda \approx 1.0)$. The different behavior between oxos and heavier analogues is interesting in connection with previous experimental and computational studies of multiple bonding involving chalcogenide complexes of the $\mathrm{Ti}$-triad metals, ${ }^{17,24}$ which suggest that oxos have a greater contribution from a singly bonded resonance structure while heavier congeners are more heavily weighted toward a doubly bonded resonance structure. The present research, covering a wide range of metals and coordination environments, suggests that this fundamental difference between multiply bonded complexes of the light main group elements and their heavier congeners may be more generally applicable than previously thought. This is an interesting and fundamental point regarding the chemistry of these important intermediates.

It is interesting that the predictive ability of the computations for vibrational frequencies is generally worse in cases where the transition metal-chalcogen bond is more polarized, e.g., first-row transition metals versus their second- and third-row congeners (Table 2) and oxos versus sulfides and selenides (Figure 4). This effect may also be operating in tetraanionic metallates, Figure 1. In other work we have shown that inclusion of monopositive counterions to model environmental effects counteracts the poorer agreement for $\left[\mathrm{M}(\mathrm{O})_{4}\right]^{4-}$ complexes. $^{21}$

As with any methodology, there are limitations to the present approach. Relative to the rest of the data, even after applying a linear correction, noticeably poorer agreement is seen for tetraanionic metallates and $\mathrm{d}^{2}$-tetraoxos. For the latter, problems may arise due to the reduction in symmetry when the energy Hessians is calculated numerically for these $T_{\mathrm{d}}$ complexes with $\mathrm{e}^{2}$ electronic ground states. Utilization of the new ECP analytic second derivatives implementation described by Russo et al. ${ }^{10}$ is therefore of even greater interest as it obviates this potential problem.

Given the limitations in theory and experiment with respect to vibrational spectra of many families of TM complexes, a good deal of care is needed to avoid attaching undue quantitative significance to vibrational data. Obvious extensions to this research include the study of new families of TM complexes, investigation of normal modes other than metal-ligand stretching, and the use of other methods, including DFT which has 
shown considerable promise. ${ }^{9,11}$ In regard to the first two points, one must contend with the limited accuracy, sensitivity to environmental factors, and ambiguity in normal mode assignment seen in the vibrational spectra of many TM complexes. An exciting application of this research is the use of frequencies estimated from theory to parameterize molecular mechanics (MM) calculations for TM complexes. MM relies on vibrational data such as force constants to parameterize the force fields. ${ }^{28}$ In general, vibrational data is sparse to nonexistent for many families of TM complexes. A reliable scheme for estimation of these data could provide the necessary parameters for MM calculations and thus overcome a primary impediment to widespread application of molecular mechanics to transition metal complexes. Initial results from this group employing this approach are encouraging. ${ }^{29}$

Acknowledgment. We greatly appreciate the assistance of Drs. John Pople and Anthony Scott in providing experimental and theoretical vibrational data for main group compounds to provide a comparison with the results of the present research. The help of Michael T. Benson, Deborah Smith, Scot Curtiss, Karen Pierce-Beaver, Hoang D. Nguyen, and Soon J. Lim (all of the University of Memphis Chemistry Department) in obtaining some of the optimized chalcogenide geometries is gratefully appreciated. Dr. Achim Müller is also gratefully acknowledged for his assistance in obtaining experimental data relevant to $\mathrm{TM}-$ chalcogenides. The support of the Air Force Office of Scientific Research, National Science Foundation (Grant CHE-9614346), American Chemical Society's Petroleum Research Fund (Grant 31070-AC3), and the Cornell Theory Center are gratefully acknowledged.

Supporting Information Available: Tables of calculated and experimental stretching frequencies for the complete collection of transition metal chalcogenides investigated as well as the scaled and linearly corrected frequencies (5 pages). Ordering information is given on any current masthead page.

\section{References and Notes}

(1) Pople, J. A.; Schlegel, H. B.; Krishnan, R.; Defrees, D. J.; Binkley, J. S.; Frisch, M. J.; Whiteside, R. A.; Hout, R. F.; Hehre, W. J. Int. J. Quantum Chem., Quant. Chem. Symp. 1981, 15, 269.

(2) Pople, J. A.; Scott, A. P.; Wong, M. W.; Radom, L. Isr. J. Chem. 1993, 33, 345 234 .

(3) Hout, R. F.; Levi, B. A.; Hehre, W. J. J. Comput. Chem. 1982, 3,

(4) Johnson, B. G.; Gill, P. M. W.; Pople, J. A. J. Chem. Phys. 1993, 7, 5612 .
(5) Rauhut, G.; Pulay, P. J. Phys. Chem. 1995, 99, 3093.

(6) Flock, M.; Ramek, M. Int. J. Quantum Chem.; Quantum Chem. Symp. 1993, 27, 331.

(7) Lee, J. Y.; Hahn, O.; Lee, S. J.; Mhin, B. J.; Lee, M. S.; Kim, K. S. J. Phys. Chem. 1995, 99, 2262.

(8) (a) Neuhaus, A.; Veldkamp, A.; Frenking, G. Inorg. Chem. 1996, 35, 5278. (b) Veldkamp, A.; Frenking, G. Chem. Ber. 1993, 126, 1325.

(9) Sosa, C.; Andzelm, J.; Elkin, B. C.; Wimmer, E.; Dobbs, K. D.; Dixon, D. A. J. Phys. Chem. 1992, 96, 6630. This work compares two DFT implementations with earlier Hartree-Fock studies using small allelectron basis sets (Dobbs, K. D.; Hehre, W. J. J. Comput. Chem. 1987, 8 , 861,880 .).

(10) Russo, T. V.; Martin, R. L.; Hay, P. J.; Rappé, A. K. J. Chem. Phys. 1995, 102, 9315.

(11) Delley, B.; Wrinn, M.; Lüthi, H. P. J. Chem. Phys. 1994, 100, 5785. Ziegler and co-workers have also reported DFT studies of TM-carbonyls, see: Bérces, A.; Ziegler, T. J. Phys. Chem. 1994, 98, 13233 and Fan, L.; Ziegler, T. J. Phys. Chem. 1992, 96, 6937.

(12) Griffith, W. P. Coord. Chem. Rev. 1970, 5, 459.

(13) Holm, R. H. Chem. Rev. (Washington, D. C.) 1987, 87, 1401.

(14) Diemann, E.; Müller, A. Coord. Chem. Rev. 1973, 10, 79.

(15) Schmidt, K. H.; Müller, A. Coord. Chem. Rev. 1974, 14, 115.

(16) Nugent, W. A.; Mayer, J. M. Metal-Ligand Multiple Bonds; Wiley: New York, 1988

(17) Benson, M. T.; Cundari, T. R.; Lim, S. J.; Nguyen, H. D.; PierceBeaver, K. J. Am. Chem. Soc. 1994, 116, 3955.

(18) Schmidt, M. W.; Baldridge, K. K.; Boatz, J. A.; Jensen, J. H.; Koseki, S.; Matsunaga, N. M.; Gordon, M. S.; Nguyen, K. A.; Su, S.; Windus, T. L.; Elbert, S. T. J. Comput. Chem. 1993, 14, 1347.

(19) Krauss, M.; Stevens, W. J.; Basch, H.; Jasien, P. G. Can. J. Chem. 1992, 70, 612

(20) Benson, M. T.; Cundari, T. R.; Li, Y.; Strohecker, L. A. Int. J. Quantum Chem., Quantum Chem. Symp. 1994, 28, 181.

(21) Simple modeling of environmental effects by the inclusion of cationic counterions brings the predicted vibrational frequencies into better agreement with experimental frequencies, see: Raby, P. D. M.S. Thesis, University of Memphis, 1996.

(22) Rabinovitch, D.; Parkin, G. J. J. Am. Chem. Soc. 1991, 113, 9421.

(23) Christou, V.; Arnold, J. J. Am. Chem. Soc. 1992, 114, 6240.

(24) (a) Parkin, G.; Howard, W. A. J. Am. Chem. Soc. 1994, 116, 606. (b) Parkin, G.; Howard, W. A. J. Organomet. Chem. 1994, 472, C1.

(25) Gardner, D. R.; Fettinger, J. C.; Eichhorn, B. W. Angew. Chem., Int. Ed. Engl. 1994, 33, 1859.

(26) Cotton, F. A.; Wilkinson, G. Advanced Inorganic Chemistry; Wiley: New York, 1988.

(27) (a) Cundari, T. R.; Benson, M. T.; Lutz, M. L.; Sommerer, S. O. In Reviews in Computational Chemistry; Boyd, D. B., Lipkowitz, K., Eds.; VCH: New York, 1996; pp 145-202. (b) Frenking, G.; Antes, I.; Böhme, M.; Dapprich, S.; Ehlers, A. W.; Jonas, V.; Neuhaus, A.; Otto, M.; Stegmann, R.; Veldkamp, A.; Vyboishchikov, S. F. In Reviews in Computational Chemistry; Boyd, D. B., Lipkowitz, K., Eds.; VCH: New York, 1996, pp 63-144.

(28) (a) Hay, B. P. Coord. Chem. Rev. 1993, 126, 177. (b) Comba, P. Coord. Chem. Rev. 1993, 123, 1. (c) Comba, P.; Hambley, T. W. Molecular Modeling of Inorganic Compounds; VCH: Weinheim, 1995.

(29) Benson, M. T.; Cundari, T. R.; Moody, E. W. J. Organomet. Chem. $1995,504,1$. 